\title{
Clinical Significance of Foxp3 Gene Expression in Patients with Lupus Nephritis
}

\author{
Amaal Abdel Aleem', Fadia Attia', Aziza Omar², Ranya Hassan ${ }^{1 *}$ \\ Departments of ${ }^{1}$ Clinical Pathology, ${ }^{2}$ Rheumatology and Rehabilitation, Faculty of Medicine, Suez Canal \\ University, Egypt
}

\begin{abstract}
Background: Lupus nephritis (LN) is an inflammation of the kidney that is one of the most serious complications of SLE. Recent studies revealed that the Foxp3 plays an important role in the pathogenesis of SLE. Objectives: To measure Foxp3 mRNA expressions in peripheral blood of patients with active and inactive form of lupus nephritis (LN) versus healthy control subjects and explore its role in disease activity. Subjects and Methods: Real time PCR was used to detect the mRNA expression of Foxp3 by SYBR Green technique in the peripheral blood mononuclear cells (PBMNCs Foxp3) of 24 patients with active lupus nephritis (LN), 10 patients with inactive lupus and 40 healthy subjects. Results: We found that Foxp 3 mRNA expression level in both patient's groups ( $n=34)$ was significantly higher than that in normal control group $(n=40),(p<0.05)$. The mRNA expression of Foxp3 in active stage $(n=24)$ was higher than that in inactive stage $(n=10)$, $(p<0.05)$. Moreover, Foxp3 mRNA level positively correlated with SLEDAI $(r=0.592, p<0.0001)$ and with serum creatinine level $(r=0.576 ; P=0.003)$. Conclusion: We concluded that PBMNCs foxp3 mRNA is markedly up-regulated in patients with active LN, and the level of expression is closely correlated with the clinical disease activity. Measurement of Foxp3 mRNA in peripheral blood may be a non-invasive risk biomarker for assessing the disease severity in $\mathrm{LN}$.
\end{abstract}

Key words: $C D 4^{+} \mathrm{CD} 25^{+}$Tregs, Foxp3 mRNA, SLE

\section{Introduction}

Lupus nephritis (LN) is one of the most critical complications of systemic lupus erythematosus (SLE), as about $60 \%$ of SLE patients can show this end-organ involvement ${ }^{(1,2)}$. $L N$ develops early in the sequence of SLE therefore becoming a major predictor of poor prognosis. LN treatment is considered a great challenge because of its unpredictable course. However, clinical outcome of renal involvement can be noticeably improved by early diagnosis(3). The pathogenesis of lupus nephritis remains an active field of study. T regulatory cells (Tregs)
Tregs are a specialized subset of T cells that function to control the immune response. Tregs might also play a critical immunosuppressive function in SLE as well as in LN. Altered wide variety of Foxp3-positive cells in SLE was proven, and expression of Foxp 3 is probably related to the activation of $C D 4+T$ cells induced through disease activity ${ }^{(4-6)}$. Forkhead box protein $\mathrm{P}_{3}$ (Foxp3), is a member of the functionally diverse Forkhead/winged helix own family of DNA binding transcription factors( $(7)$, its expression seems to be a greater sensitive parameter that discriminates among activated $\mathrm{CD} 4{ }^{+} \mathrm{CD} 25^{\text {low }} \mathrm{T}$ effector cells and $\mathrm{CD}_{4}{ }^{+}$ 
CD25 $5^{\text {high }}$ Treg cells. Research studies confirmed that some adaptive Treg cells lose their $C_{2} 25$ expression in vivo but maintain FoxP3 expression ${ }^{(8)}$, so it is a marker with greater sensitivity for Treg identification ${ }^{(9-}$ 12). Gold standard diagnosis for LN is kidney biopsy. In addition of being an invasive technique, sampling bias during biopsy is a real problem as kidney affection in LN is not uniformal. From this point of view, searching for biomarkers that can be used as an immediate, noninvasive indicator of kidney status and hence a reflection of LN activity is ongoing(13). Urinary expression of Foxp3 has recently been detected in LN patients and has been suggested to be a biomarker for assessing disease activity, but unfortunately it is very expensive and cannot be used as a routine checkup investigation, moreover the cellular origin of Foxp3 mRNA expression in the urine is still not confirmed and hence correlation between intra-renal Foxp3 mRNA and urinary results is still undetermined $^{(14)}$. From this point of view, we quantified the peripheral blood mononuclear cells Foxp3 mRNA gene expression in patients with $\mathrm{LN}$ and investigated whether the expression is related to disease activity and whether it can be used as a diagnostic marker for LN.

\section{Patients and Methods}

A case-control study design was used to investigate the levels of Foxp 3 mRNA in peripheral blood mononuclear cells (PBMNCs) as a non-invasive biomarker for assessing the activity of LN and SLE. This study was performed at Clinical Pathology, Nephrology, Rheumatology and Rehabilitation Departments of Suez Canal University Hospital, Ismailia, Egypt.

Study Population:

Biopsy - proven lupus nephritis patients diagnosed at LN Clinic in Suez Canal University Hospital, Ismailia were included in the study. The study included 2 groups of patients: Group 1 (The Active Group): Twenty-four active LN patients. All patients were diagnosed according to the American
College of Rheumatology (ACR) diagnostic criteria ${ }^{(15)}$ and active $L N$ was defined as the systemic lupus erythematosus disease activity index (SLEDAI) score $\geq 6$. The renal disease activity was assessed clinically by the SLEDAI renal score which derives from the total SLEDAI score. The overall SLEDAI score ranges from 0 to 24 while SLEDAI renal score ranges from 0 to 16 . The SLEDAI renal score consists of Hematuria, Proteinuria, Pyuria and Urinary red cell casts. Hematuria and pyuria defined as more than 5 cells per high power field and proteinuria was defined as a urine protein/Creatinine ratio $0.3 \mathrm{~g} / \mathrm{g}$ creatinine. The activity index of lupus nephritis in kidney biopsies was defined by the sum of semi quantitative scores of six lesions including: Hypercellularity, Leukocyte infiltration, Sub endothelial hyaline deposits, Interstitial inflammation, Necrosis and Cellular crescents. Each lesion is scored o3 and the last two items are scored twice. The maximum of activity index is 24 points. Group 2 (The Inactive Group): Ten patients with history of type III or IV LN whose disease became quiescent for at least 6 months after treatment. Group 3 (The Control Group): Forty age and sex matched healthy individuals were studied as controls. Exclusion criteria: We excluded patients with Life-threatening complications other than lupus nephritis (for example, cerebral lupus, severe systemic infection), other autoimmune disease and pregnant patients. Data was collected from LN patients using the followings: A) Interview Questionnaire: Subjects were interviewed using a questionnaire about the following data: - Personal history: name, age, sex, address, history of other autoimmune diseases (for example, rheumatoid arthritis). Clinical data including SLEDAI, proteinuria and serum creatinine were recorded. B) Clinical Examination: General examination includeing: general appearance, vital signs, head, 
neck, heart, chest, and abdominal examination. C) Laboratory Investigations: Sampling: Peripheral venous blood $(2 \mathrm{ml})$ was collected by venipuncture on EDTA tube under sterile condition, with informed consent from all study subjects. Total RNA extraction and reverse transcription: Total RNA was extracted from EDTA blood by the usage of the RNeasy Mini package (QIA amp minikit, Qiagen, US) using the following steps: Lysis of erythrocytes, Lysis of leukocytes, Selective binding of the genomic DNA to a specific carrier and separation of contaminating DNA on the surface of the DNA binding mineral carrier particles, transferring of the remaining sample into the RNA binding RTA Spin Filter, followed by the adjustment of the binding conditions, binding of the total RNA to the membrane, while proteins remain in the filtrate, washing of the membrane and elimination of contaminants and ethanol. Elution of highly pure total RNA: After that C-DNA was synthesized by incubating $10 \mu$ l total RNA (incorporate up to $1 \mu \mathrm{g}$ RNA) in a $20 \mu \mathrm{l}$ reaction volume consists of RT Buffer, and RT Primer mix. The whole response took place at $42^{\circ} \mathrm{C}$ and is then inactivated at $95^{\circ} \mathrm{C}$ the use of QuantiTectR opposite Transcription kit (QIAGEN, United States). Foxp3 mRNA expression: We accomplished real-time $P C R$ response to measure Foxp3 mRNA in peripheral blood following the producer's protocol. Real-time PCR was performed using a commercial kit SYBR Green QuantiTectR Primer Assay PCR master mix (Qiagen, USA) using the Step One, Applied Biosystem, Real Time Thermocycler (Chicago, United States). The threshold cycles (CT), at which an increase in reporter fluorescence above the baseline signal was first detected, were determined. GAPDH was used as endogenous control and for normalization $(\triangle C T)$ of the mRNA levels for the gene of interest. The endogenous control was subtracted from respective gene giving the $\triangle C T$ as a reflection of the relative mRNA expression. The calculations of relative gene expression were done using the $\Delta \Delta C T$ method. Because the amount of product doubles in each cycle the relative gene expression was calculated using the formula $2^{-\Delta \Delta C T}$, given in the manufacturer's instructions.

\section{Statistical analysis}

Statistical evaluation was done via SPSS version 17 (SPSS, Chicago, IL, USA). all of the outcomes are supplied as mean \pm S.D. Annova test and Mann-Whitney U-test were used to examine gene expression stages between groups and clinical parameters. A p-value of $<0.05$ was considered statistically significant.

\section{Results}

Table (1) shows the clinical and laboratory characteristics of the active, inactive lupus groups and healthy control groups. The table shows mean serum creatinine, protein in 24 hours' urine collection and mean SLE disease activity index. Table (2) and figure 1 show the mean levels of Foxp 3 among active and inactive lupus group. Active lupus group had statistically significant higher levels of Foxp3 than inactive group (21.3 versus 3.6 respectively) ( $p<0.001$ ). Table (3) shows the correlations between levels of Foxp3 and the studied variables. There was a significant positive correlation between higher levels of Foxp3 and higher mean serum creatinine level (figure 2) $(r=0.576, p=0.003)$, higher mean SLEDAI values $(r=0.934, p<0.0001$, figure 3$)$. 

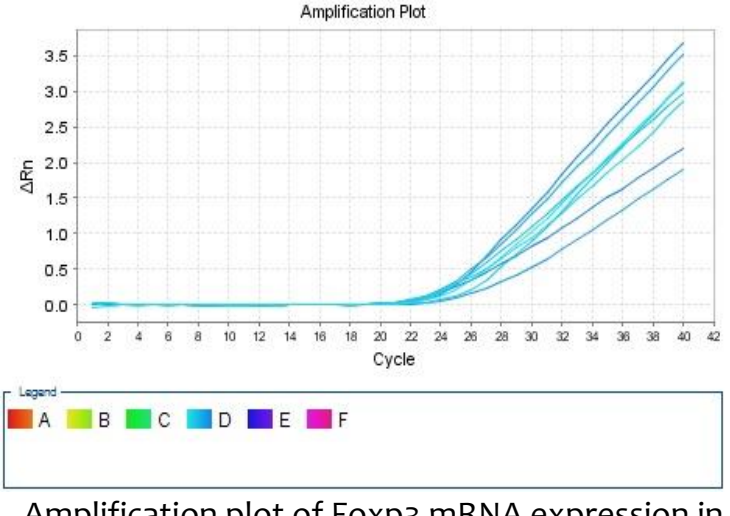

Amplification plot of Foxp3 mRNA expression in patients

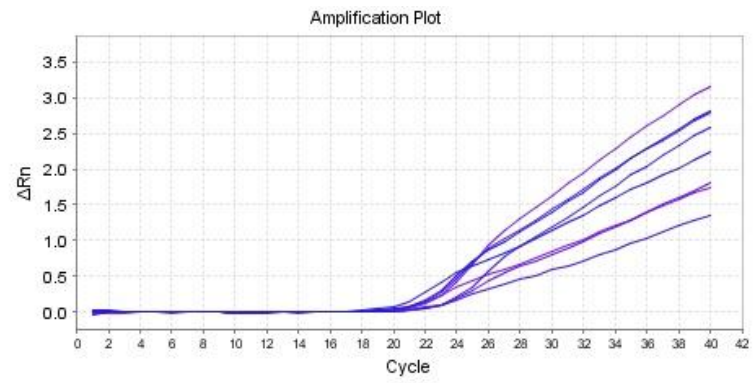

$A \quad B \square C \square D \square E \square F$

Amplification plot of GAPDH in patients

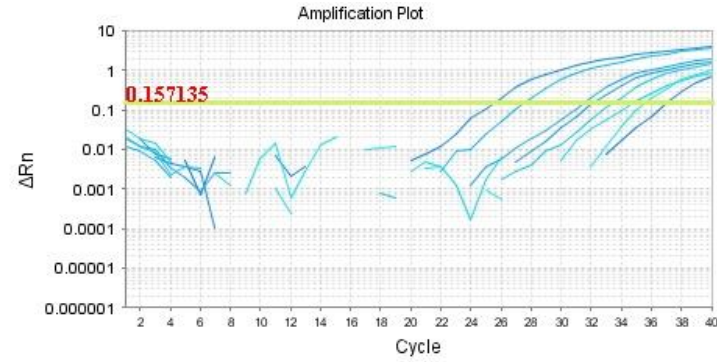

L Legent $\mathrm{B} \square \mathrm{C} \square \mathrm{D} \square \mathrm{E}$

Amplification plot of Foxp3 with baseline threshold

\section{Discussion}

The goal of this study was to assess the levels of Foxp3 mRNA in peripheral blood of patients with lupus nephritis as a non-invasive biomarker for assessing the activity of LN. In the present study, we studied PBMNCs mRNA expression of Foxp3 in patients with LN for the first time to our knowledge. We found that Foxp3 mRNA

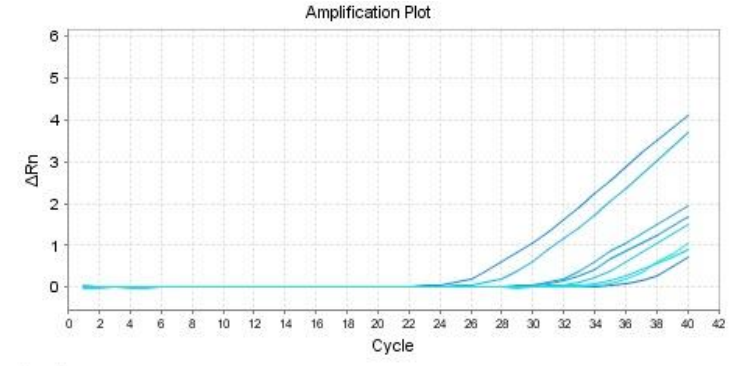

$[\mathrm{A} \square \mathrm{B} \square \mathrm{C} \square \mathrm{D} \square \mathrm{F}$

Amplification plot of Foxp3 mRNA expression in normal controls

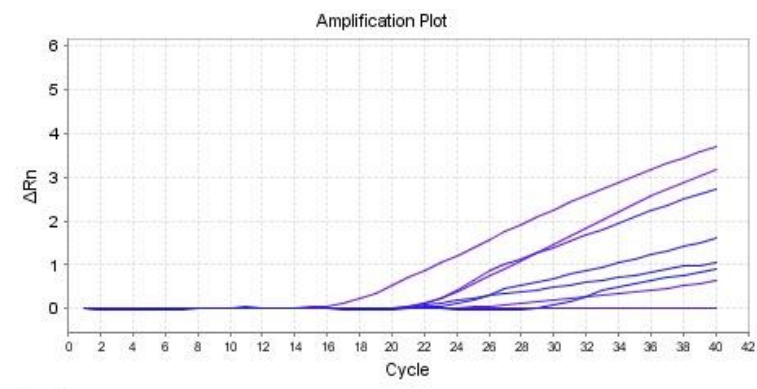

$\square \square C \square D \square E \square$

Amplification plot of GAPDH in normal controls

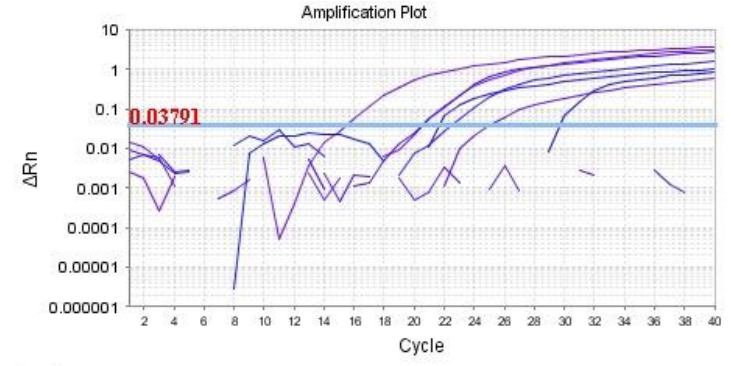

$[\mathrm{A} \square \mathrm{B} \square \mathrm{C} \square \mathrm{D} \square \mathrm{E}$

Amplification plot of GAPDH with baseline threshold

was higher in patients with active lupus compared to those with silent lupus and PBMNCs Foxp3 mRNA significantly correlated with SLEDAI and creatinine level. Our results suggested that a higher Foxp3 mRNA, indicating a higher activity of Tregs, is related to more severe $L N$. However recent studies showed that number of natural Tregs in patients with active SLE was decreased in comparison to patients with 


\begin{tabular}{|c|c|c|c|c|}
\hline Variables & $\begin{array}{c}\text { Active LN } \\
(n=24)\end{array}$ & $\begin{array}{l}\text { Inactive LN } \\
\qquad(\mathrm{n}=10)\end{array}$ & $\begin{array}{c}\text { Control } \\
\text { Group }(n=40)\end{array}$ & $p$ value \\
\hline $\begin{array}{l}\text { Age (years) } \\
\text { Range }\end{array}$ & $\begin{array}{c}35 \cdot 4 \pm 6.4 \\
23-44\end{array}$ & $\begin{array}{c}39.7 \pm 7.9 \\
23-49\end{array}$ & $\begin{array}{c}32.4 \pm 6.1 \\
25-41 \\
\end{array}$ & 0.11 \\
\hline Gender (Male/ Female) & $0 / 24$ & $1 / 9$ & $0 / 10$ & - \\
\hline Creatinine mg/dl & $1.7 \pm 0.45$ & $0.9 \pm 0.6$ & - & 0.67 \\
\hline Protein in urine $(\mathrm{g} / \mathrm{l})$ & $2.5 \pm 2.0$ & $0.7 \pm 0.4$ & - & *0.001 \\
\hline SLEDAI Mean \pm SD & $9.8 \pm 3.7$ & $1.2 \pm 0.8$ & - & *0.001 \\
\hline
\end{tabular}

Data are presented as Mean $\pm \mathrm{SD}$; $\mathrm{LN}=$ lupus nephritis; ${ }^{*} p$ value significant Difference between Active and Inactive Groups (by Mann-Whitney U-test)

\begin{tabular}{|c|c|c|c|}
\hline Variables & $\begin{array}{c}\text { Active LN } \\
(n=24)\end{array}$ & $\begin{array}{c}\text { Inactive } \\
\text { LN }(n=10)\end{array}$ & $p$-value \\
\hline $\begin{array}{l}\text { FOXP3 } \\
\text { Mean } \\
\pm \text { SD } \\
\text { Range }\end{array}$ & $\begin{array}{c}21.3 \pm 13.2 \\
8-53\end{array}$ & $\begin{array}{c}3.6 \pm 1.3 \\
2-6\end{array}$ & $0.002 * *$ \\
\hline
\end{tabular}

$\mathrm{LN}=$ Lupus nephritis; **Highly significant $p$ value $<0.001$

Table 3: Correlations between levels of Foxp3 mRNA expression and the studied variables

\begin{tabular}{|l|l|l|}
\hline & \multicolumn{2}{|l|}{ Foxp3 mRNA } \\
\cline { 2 - 3 } & $\begin{array}{l}\text { Correlation } \\
\text { Coefficient }\end{array}$ & p-value \\
\hline Age & 0.060 & 0.780 \\
\hline Biopsy grade & 0.153 & 0.476 \\
\hline Serum creatinine & 0.576 & $0.003^{* *}$ \\
\hline Protein in urine & 0.041 & 0.850 \\
\hline SLEDAI & 0.934 & $<0.0001^{* *}$ \\
\hline
\end{tabular}

**Correlations are highly significant at $p$-value $<0.01$

inactive lupus or normal subjects, and the reduced percentage of circulating Tregs inversely correlated with lupus disease activity ${ }^{(16,17)}$. A higher mRNA expression of Foxp3 in blood T cells was observed in patients with active SLE ${ }^{(18)}$. Moreover, urinary Foxp3 mRNA was found to be higher in patients with active lupus compared to those with silent lupus and urinary Foxp3 mRNA and significantly correlated with SLEDAI and proteinuria ${ }^{(14)}$. This apparent contradiction can be due to the expression of
$\mathrm{FOXP}_{3}$ from $\mathrm{CD}_{4}{ }^{+} \mathrm{T}$ cells as well as natural Tregs during activation in active $\operatorname{SLE}^{(19,20)}$ although it is still debatable whether this activation-induced Foxp3 mRNA indicates a functional Treg capacity or not. Another possibility is the activation of destructive effector cells as well as protective Tregs during the active stage of LN, which therefore results in activation-induced Foxp3 $\mathrm{mRNA}^{(21)}$. Moreover, recent mice and human studies have demonstrated that Treg cells can be reprogrammed into a novel population, IL-17+Foxp3+T cells, phenotypically and functionally resembling Th17 cells under the complicated cytokine stimulation which could be one of the explanation for increased level of Foxp3 expression in active $\mathrm{LN}^{(22)}$. In summary, our result is in line with the above observations. Taken together, presented evidences suggest that the overall expression of Foxp3 is increased in active $L N$, but the suppressive function of $\mathrm{CD}_{4}{ }^{+} \mathrm{CD} 25^{+}$Tregs may be defective. Our results were in agreement with Abbass et al ${ }^{(23)}$ who found increased Foxp3 expression in active SLE patients in comparison to inactive, Lee et al ${ }^{(18)}$ also found increased Foxp3 gene expression in active SLE disease while Yan et al ${ }^{(24)}$ found no difference in Foxp3 expression at either the mRNA or protein level in any CD4+, CD25+ $T$ cell subset from SLE patients as compared with controls. Alvarado Sanchez et 
al $^{(25)}$ also reported a significant inverse correlation between disease activity of SLE and the suppressive function of Treg. These discrepancies may arise from fair dif-

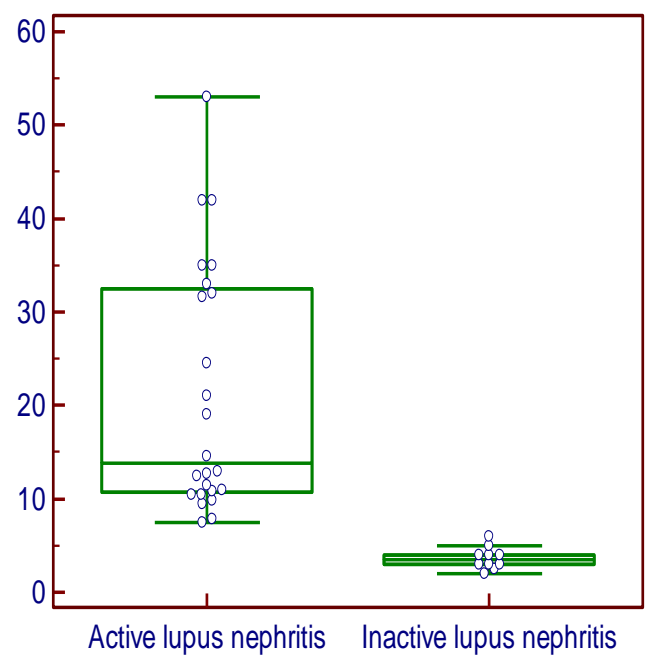

Figure 1: mRNA Level of Foxp3 of the studied patients with active and inactive $\mathrm{LN}$ represented by $25^{\text {th }}, 50^{\text {th }}$ (median), $75^{\text {th }}$ percentiles, minimum, and maximum

ferences between isolation protocols, flow-cytometry or RT-QPCR technicalities resulting in strong difficulties in comparing results from different studies. Although we found a significant positive correlation between higher levels of Foxp3 mRNA and higher mean serum creatinine levels $(r=0.576, p<0.003)$, higher mean SLEDAI values $(r=0.934, p<0.0001)$, and higher ac-

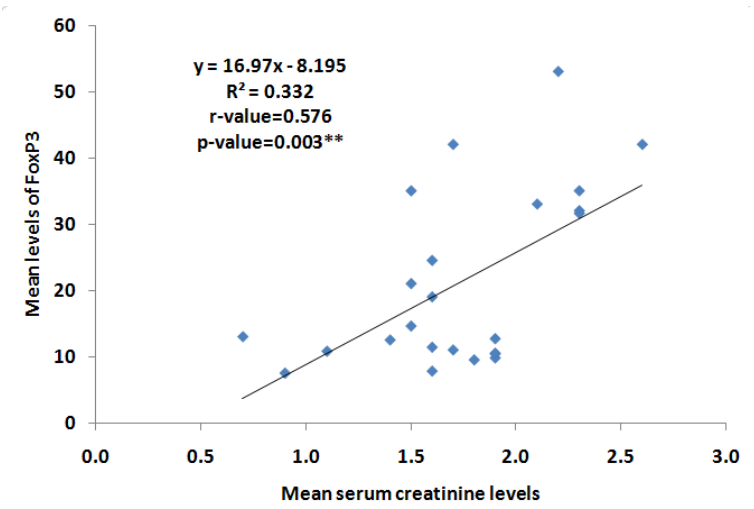

Figure 2: Significant positive correlation between level of Foxp3 and serum creatinine level $(r=0.576, p=0.003)$ tivity ( $r=0.592, p<0.0001)$, we did not find any correlation between Foxp 3 gene expression and proteinuria. However, Bonelli et al (26) showed that the increase of Foxp $3+T$ cells is linked to active nephritis as well as a correlation between Foxp3+ cells and the extent of proteinuria further support the idea of Foxp3+ cells as a marker to recognize and monitor patients with renal involvement. This discrepancy could be explained that proteinuria may not necessarily indicate ongoing inflammation in the kidneys and may be contributed by pre-existing chronic lesions or recent damage in the kidneys during the course of the disease.

\section{Conclusion}

peripheral blood mRNA expression of Foxp3 is markedly up-regulated in patients with active LN and the level of Foxp3 mRNA significantly correlates with disease activity. These preliminary results suggest that urinary mRNA expression of Foxp3 might be used as a risk biomarker for assessing disease activity However, future studies are needed to define the role of this marker.

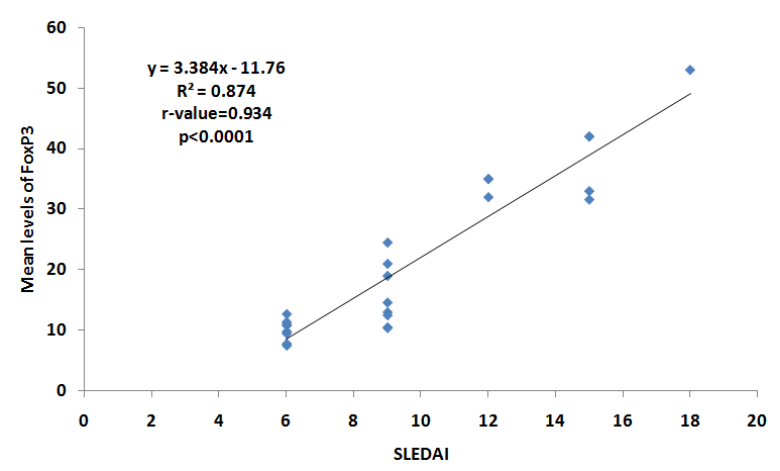

Figure 3: Significant positive correlation between level of Foxp3 and SLEDAI index value $(r=0.934, p<0.0001)$. 


\section{References}

1. Bertsias G, Gordon C, Boumpas DT. Clinical trials in SLE: lessons learned from the past as we recede to the future - the EULAR recommendations for the management of SLE and the use of end-points in clinical trials. Lupus. 2008; 17:437-442.

2. Tucci M, Stucci S, Strippoli S, Silvestris F. Cytokine overproduction, T-cell activation, and defective T-regulatory functions promote nephritis in systemic lupus erythematosus. J Biomed Biotechnol. 2010:457146.

3. Anaya JM, Cañas C, Mantilla RD. Lupus nephritis in Colombians: contrasts and comparisons with other populations. Clin Rev AllergImmunol. 2011;40(3): 199-207.

4. Lewis EJ, Schwartz MM. Pathology of lupus nephritis. Lupus. 2005;14(1):3138.

5. Oates JC, Varghese S, Bland AM, Taylor TP, Self SE, Stanislaus R, et al. Prediction of urinary protein markers in lupus nephritis. Kidney Int. Dec;2005; 68(6):2588-92.

6. Cross J, Jayne D. Diagnosis and treatment of kidney disease Best Pract Res ClinRheumatol.2005 19:785-98.

7. Banham AH, Powrie FM, Suri-Payer E. FOXP3 regulatory $T$ cells: current controversies and future perspectives. Eur $\mathrm{J}$ Immunol 2006; 36:2832-6.

8. Fontenot JD, Gavin MA, Rudensky AY. Foxp3 programs the development and function of $\mathrm{CD} 4+\mathrm{CD} 25+$ regulatory $\mathrm{T}$ cells. Nat Immunol .2003; 4, 330-336.

9. Tran DQ., Ramsey H, Shevach EM. Induction of FOXP3 expression in naive human CD4 + FOXP3- T cells by T cell receptor stimulation is TGF $\{$ beta\}-dependent but does not confer a regulatory phenotype. Blood .2007; 110, 2983-2990.

10. Allan SE, Crome SQ, Crellin NK, Passerini L, Steiner TS., et al. Activation-induced FOXP3 in human T effector cells does not suppress proliferation or cytokine production. IntImmunol.2007; 19, 345-354.

11. Pillai V, and Karandikar NJ. Human regulatory T cells: $A$ unique, stable thymic subset or a reversible peripheral state of differentiation? Immunology Letters .2007; 114, 9-15.

12. Li, S., Gowans, E. J., Chougnet, C., Plebanski, M., Dittmer, U. Natural regulatory $T$ cells and persistent viral infection. J Virol .2008 82, 21-30.

13. de Zubiria Salgado A, Herrera-Diaz C. Lupus nephritis: an overview of recent findings. Autoimmune Dis. 2012: 8496 84.

14. Wang G., Lai F.M., Tam L.S., Li E.K., Kwan B.C., Chow K.M., et al. Urinary FOXP3 mRNA in patients with lupus nephritis-relation with disease activity and treatment response. Rheumatology (Oxf). 2009; 48: 755- 60.

15. Hochberg MC. Updating the American College of Rheumatology revised criteria for the classification of systemic lupus erythematosus, Arthritis Rheum. 1997; 40: 1725-6.

16. Bonelli M., Savitskaya A., Steiner C.W., Rath E., Smolen J.S., and Scheinecker C., Phenotypic and functional analysis of CD4+ CD25- Foxp3+ $T$ cells in patients with systemic lupus erythematosus. J Immunol. 2009; 182: 1689-95.

17. Crispin JC, Martínez A, Alcocer-Varela J. Quantification of regulatory T cells in patients with systemic lupus erythematosus. J Autoimmun. 2003;21(3):273-6.

18. Lee J-H, Wang L-C, Lin Y-T, Yang Y-H, Lin D-T, Chiang B-L. Inverse correlation between $\mathrm{CD} 4^{+}$regulatory $\mathrm{T}$-cell population and autoantibody levels in pediatric patients with systemic lupus erythematosus. Immunology. 2006;117 (2):280-286.

19. Walker M, Kasprowicz DJ, Gersuk VH, et al. Induction of $\mathrm{FoxP}_{3}$ and acquisition of $\mathrm{T}$ regulatory activity by stimulated human $\mathrm{CD} 4^{+} \mathrm{CD} 25^{-} \mathrm{T}$ cells. Journal 
of Clinical Investigation. 2003; 112(9):

1437-1443.

20. Morgan $M$, van Bilsen $\mathrm{JH}$, Bakker $\mathrm{AM}$, et al. Expression of FOXP3 mRNA is not confined to $C D 4+C D 25+T$ regulatory cells in humans, Hum Immunol, 2005; 66 :13-20.

21. Muthukumar T, Dadhania D, Ding R, Snopkowski $C$, et al. Messenger RNA for FOXP3 in the urine of renal-allograft recipients. N Engl J Med. 2005 1;353 (22):2342-51.

22. Du R, Zhao H, Yan F, Li H. IL-17+Foxp3+ $T$ cells: an intermediate differentiation stage between Th17 cells and regulatory T cells. J Leukoc Biol. 2014;96 (1): 39-48.

23. Abbass AA, Mohamed NA, Abdel-Rehim AS. Association of Foxp3 regulatory gene expression with systemic lupus erythematosus disease activity among Egyptian patients. Egypt J Immunol. 2013;20(2):21-8.

24. Yan B, Liu Y. The nature of increased circulating CD4+ CD25-Foxp3+ T cells in patients with systemic lupus erythematosus: a novel hypothesis. The open rheumatology journal. 2009;3(1):22-4.

25. Alvarado-Sanchez B, Hernandez-Castro $B$, Portales-Perez $D$, et al. Regulatory $T$ cells in patients with systemic lupus erythematosus. J Autoimmun. 2006; 27:110-118.

26. Bonelli $M$, Göschl L, Blüml S, Karonitsch T, Steiner CW, et al. CD4 CD25- Foxp3 T cells: a marker for lupus nephritis? Arthrit Res Ther. 2014;16(2): R104 